\title{
Superficial Mycoses in Menopausal Age Women: 21 years' Experience
}

\section{Arenas R*, Guevara-Cervantes Javier F, Perez-Rojas Diego O, and Vasquez-del-Mercado E}

Mycology Section, Dr. Manuel Gea Gonzalez General Hospital, Mexico

*Corresponding author: Roberto Arenas, Mycology Section, Dr. Manuel Gea Gonzalez General Hospital, Mexico, Tel: 56156884; E-mail: rarenas98@hotmail.com

Received date: May 31, 2016; Accepted date: June 29, 2016; Published date: June 30, 2016

Citation: Arenas R (2016) Superficial Mycoses in Menopausal Age Women: 21 years' Experience. Med Mycol Open Access 2: 4. doi: $10.21767 / 2471-8521.100015$

Copyright: (c) 2016 Arenas R, et al. This is an open-access article distributed under the terms of the Creative Commons Attribution License, which permits unrestricted use, distribution, and reproduction in any medium, provided the original author and source are credited.

\section{Abstract}

Background: Menopause is the permanent and irreversible absence of menstruation for at least a year. Usually, it is accompanied by typical physical and mental symptoms. Although, infections during menopause have been extensively documented, there is a lack of information regarding superficial mycoses.

Objective: Our objective was to determine the frequency of superficial mycoses in menopausal age women.

Methods: This is a retrospective chart review of menopausal age patients seen at the Mycology section in a single medical institution in Mexico City from 1994 to 2014. Clinical, epidemiological, and mycological data were reviewed.

Results: There were 881 patients studied and 811 cases were confirmed by a mycological test. Diagnoses in menopausal age women included onychomycosis (84.5\%), tinea pedis $(25.6 \%)$, candidiasis $(7.8 \%)$, and tinea corporis (4.3\%). The most common presentations of onychomycosis were distal and lateral subungueal (29.7\%) and total dystrophic (32.2\%). In $36.7 \%$ the diagnosis was simply "onychomycosis". The most common etiologic agents in onychomycosis were Trichophyton rubrum (39.8\%) and Candida spp. (45.3\%). The most common etiologic agent in tinea corporis and pedis was T. rubrum. The types of candidiasis were interdigital $(29.6 \%)$, oral $(45.3 \%)$, and intertrigo (17.1\%). The most common isolates were Candida spp. (55.5\%) and C. albicans (29.6\%).

Conclusions: The most common superficial mycoses in menopausal age are onychomycosis and tinea pedis, followed by tinea corporis and candidiasis. Their frequency is similar to that seen in geriatric patients and comorbidities as diabetes and hypertension.

\section{Keywords Menopause; Superficial mycoses;} Dermatophytes; Tinea pedis; Tinea corporis; Onychomycosis; Candidiasis

\section{Introduction}

Menopause is the permanent cessation of menstruation for at least 1 year. It is due to declining ovarian function and estrogen deficiency [1]. In most women, menopause occurs at 50-55 years of age, although this is extremely variable. Premature (4th decade of life) and late (7th decade) onsets are not rare [2]. It is of interest that very little is known about cutaneous infections by dermatophytes and Candida spp during menopause. Herein, we will describe the frequency during a 21-year- period at a single teaching institution.

\section{Materials and Methods}

Retrospective chart review of menopausal age patients documenting frequency of types and agents of superficial mycosis (SM) in women at the Mycology Section at "Dr. Manuel Gea Gonzalez, General Hospital" in Mexico City, from 1994 to 2014.

Menopause was defined as the absence of menstruation for at least 1 year and diagnosis of SM was established by a positive $\mathrm{KOH} /$ Chlorazol Black $\mathrm{E}^{\circledR}$ (Delasco, Council Bluffs, LA). Clinical and epidemiological data were gathered in all cases including age, occupation, date of service, co-morbidities and their treatment, clinical presentation, and mycological study. The latter included $\mathrm{KOH}$ / Chlorazol Black tests and culture was done on Sabouraud and/or Sabouraud agar with chloramphenicol-actidione (Mycocel ${ }^{\circledR}$, Difco, Ann Arbor, MI, USA). For all positive cultures, we performed macro-and microscopic identification of colonies and classification of etiologic agents. When an opportunistic fungus was identified, it was isolated at least 3 times. If a yeast was isolated a commercially chromogenic Candida speciation media was used (CHROMagar (C), Becton Dickinson co, Heidelberg, Germany), incubated at $37^{\circ} \mathrm{C}$ for 48 hours and following manufacturer's instructions for identification (C. glabrata, C. kruzei, C. albicans and C. tropicalis).

\section{Results}

In our Institution at the Mycology Section 881 patients were studied and there were 811 menopausal age women with 
confirmed SM. 182 patients (22.44\%) of these confirmed cases had more than one SM. All of them were between $50-55$ years of age. The most common diagnoses were: onychomycosis in
686 cases $(84.5 \%)$, tinea pedis in $208(25.6 \%)$, candidiasis in 64 $(7.8 \%)$, and tinea corporis in 35 (4.3\%) (Figure 1).

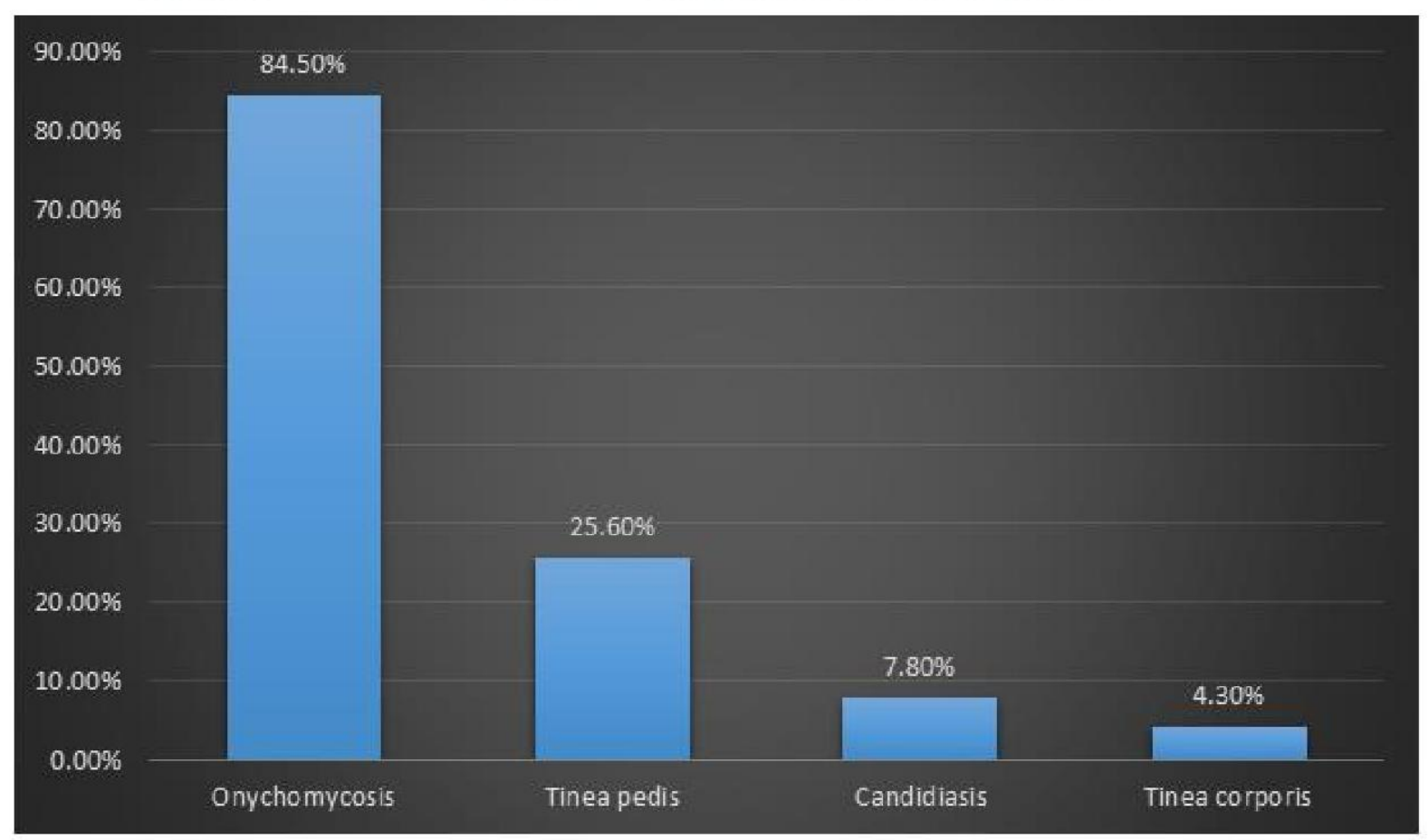

Figure 1: Superficial mycoses in menopausal age women from 1994 to 2014.

We documented the next occupations: Housewives $(72.1 \%)$, merchants $(9.8 \%)$, employees $(8.8 \%)$, graduates $(8.3 \%)$, and others $(0.7 \%)$. The most common co-morbidities were high blood pressure in 142 patients (17.5\%) and diabetes in 141 (17.3\%).

\section{Onychomycosis}

There were 686 cases. The clinical presentation was not documented in 252 cases (36.7\%). These cases were labeled "onychomycosis" only. There were 204 cases of distal lateral subungual onychomycosis (29.7\%), 221 cases of total dystrophic onychomycosis (32.2\%), 5 of white superficial onychomycosis $(0.7 \%), 2$ of proximal white subungual onychomycosis $(0.2 \%)$, and 2 cases of melanonychia $(0.2 \%)$. We found in $18 \%$ association with tinea pedis.

The most common etiologic agents in onychomycosis were Trichophyton rubrum (39.8\%) and Candida spp. (45.3\%). Less commonly, we isolated $T$ mentagrophytes (1\%), E floccosum $(0.01 \%)$, and $M$ canis (0.01\%). We isolated 12 non dermatophyte molds and the most frequent was Acremonium spp $(<1 \%)$, as we mentioned before and in order to confirm the pathogenic role, these opportunistic fungi were isolated by culture at least three times (Table 1).

Table 1: Oportunistic fungi (non dermatophytes molds) isolated in menopausal age women with onychomycosis.

\begin{tabular}{|c|c|c|}
\hline Oportunistic fungi & 12 Cases & \% \\
\hline Acremonium spp. & 1 & 24.99 \\
\hline Fusarium spp. & 5.33 & 41.65 \\
\hline Aspergillus spp. & 2 & 16.66 \\
\hline Scopulariopsis spp. & 1 & 8.33 \\
\hline
\end{tabular}




\section{Tinea pedis}

There were 208 cases. The most common etiologic agents were $T$. rubrum in 41 (83.6\%), T. mentagrophytes in 6 (12.2\%), E. floccosum in $1(2 \%)$, and $M$. canis in $1(2 \%)$. Plantar and interdigital involvement was observed in 204 cases and the vesiculobullous type in only 4 cases caused by $T$. mentagrophytes.

\section{Candidiasis}

There were 64 cases. The most common presentations were oral candidiasis in 29 (45.3\%), interdigital in 19 (29.6\%), skin folds in $11(17.1 \%)$, periungual in $2(3.1 \%)$, and vaginal in 3 (4.6\%). The most common etiologic agents were $C$ albicans in 8 (29.6\%), C krusei in 2 (7.4\%), C tropicalis in 2 (7.4\%) and Candida speciation was not confirmed in 15 (55.5\%). One out of every 3 cases, had diabetes mellitus.

\section{Tinea corporis}

Of the 35 cases of tinea corporis, all with a positive $\mathrm{KOH}, 23$ (65.7\%) were affecting the trunk, buttocks, proximal extremities or face; 6 (17.1\%) on the groin, and $6(17.1 \%)$ on hands. The most common etiologic agents were $T$. rubrum in $12(85.7 \%)$ and $M$. canis in 2 cases with trunk and hand involvement (14.2\%).

\section{Discussion}

There is an abundance of data documenting the immunologic changes and deficiencies that occur during menopause, including sex-specific mortality and infection rates. In general, men are more susceptible to certain infections by bacteria, viruses, fungi, and parasites. Testosterone is immunosuppressive, and increases severity of tuberculosis, amebiasis, leishmaniasis, and malaria.

On the other hand, normal estrogen levels in premenopausal females lead to lower mortality from pneumococcal, meningococcal, and other infections (except sexually transmitted diseases and vulvovaginal candidiasis) [3] For example, in the USA, bacterial vaginosis was documented in $23-38 \%$ of postmenopausal women. In contrast, Candida was present in only $5-6 \%$ of these patients, and decreased by $7 \%$ with each year of age [4]. In Italy, T. vaginalis or C. albicans vaginal infection was made in one-third of menopausal patients [5].

It is important to mention that skin diseases and especially $\mathrm{SM}$ in this age group can be related not only with menopause, but also with elderliness and other comorbidities as diabetes mellitus.

We report 811 menopausal women with SM. The most common diagnoses were onychomycosis (84.5\%), tinea pedis $(25.6 \%)$, candidiasis (7.8\%), and tinea corporis (4.3\%).

Frequency of onychomycosis in our patients is higher than the literature suggests. In a study of people older than 60 years in Spain the incidence of onychomycosis was 21\% [6].
Other studies have demonstrated that the incidence of onychomycosis increases with age: $40 \%$ in patients between $60-70$ years old and $61.5 \%$ after 80 years of age [7]. The incidence also varies according to geographic location: Europe (22-26\%), and Guatemala (65\%) [8]. In our series, the most common etiologic agent was T. rubrum followed by Candida spp., and T. mentagrophytes. Clinically, the most common presentations were distal and lateral subungual and total distrophic onychomycosis.

We found a similar frequency of tinea pedis (25.6\%) to previous reports: $23 \%$ in patients older than 60 years of age in Spain [6], and 22-26\% in Canada and Germany [7]. We also found as the most common etiologic agent T. rubrum followed by $T$. mentagrophytes.

As regards Candida infections, we found 3 predominant clinical presentations, including interdigital, oral, and intertriginous affection. The most common etiologic agent was C. albicans and C. krusei but Candida speciation was not confirmed in 15 (55.5\%). The numbers were too small to draw significant conclusions, [4] and the literature regarding cutaneous candidiasis in menopause is very scarce. Most research has focused on mucosal infections. For example, in Italy $T$. vaginalis or $C$. albicans vaginal infection was found in one-third of menopausal patients [5].

Our cases include 35 patients with diagnosis of tinea corporis. The most common location was the trunk followed by buttocks, proximal extremities and face. The most frequent etiologic agents were T. rubrum and $M$. canis.

In sum, we have described a large series of menopausal women with documented cutaneous infections due to dermatophytes and Candida. The most interesting finding is an increased frequency of onychomycosis. The reasons for this increase are unknown but may be due to the various immunologic and endocrine changes that occur during menopause and aging. After menopause, there is an increase in several pro-inflammatory cytokines such as TNF-alpha, IL-6, and IL-1. Also, there is an enhanced response of immune cells to these molecules, a decrease in lymphocytes (B and CD4 T), and a weaker cytotoxic activity of NK cells. All these changes have been attributed to estrogen deprivation [9].

Additionally, in this age group, the skin suffers significant changes. There is dermal atrophy and decreased blood vessels, mast cells, and fibroblasts. The result is a thinner, dryer, and more fragile skin that is more prone to infection, trauma, and bleeding $[10,11]$. All these changes would suggest and explain an increased risk of infection by dermatophytes and Candida.

Limitations in our study include its retrospective nature and the lack of positive cultures in many cases. In addition, this paper is a purely descriptive study. Also, results from this Mexican cohort cannot be extrapolated to other populations.

\section{Reference}

1. Kuhle BX (2007) An evolutionary perspective on the origin and ontogeny of menopause. Maturitas 57: 329-337. 
2. Meeta, Digumarti L, Agarwal N, Vaze N, Shah R, et al. (2013) Clinical practice guidelines on menopause: An executive summary and recommendations. J Midlife Health 4: 77-106.

3. Giefing-Kroll C, Berger P, Lepperdinger G, GrubeckLoebenstein B (2015) How sex and age affect immune responses, susceptibility to infections, and response to vaccination. Aging Cell 14: 309-321.

4. Hoffmann JN, You HM, Hedberg EC, Jordan JA, McClintock MK (2014) Prevalence of bacterial vaginosis and Candida among postmenopausal women in the United States. J Gerontol B Psychol Sci Soc Sci 69: S205-S214.

5. Spinillo A, Bernuzzi AM, Cevini C, Gulminetti R, Luzi S, et al. (1997) The relationship of bacterial vaginosis, Candida and Trichomonas infection to symptomatic vaginitis in postmenopausal women attending a vaginitis clinic.Maturitas 27: 253-260.

6. Lázaro D, Guillen F, Novel V (2004) Pensando en los pies. Piel 19: 184-190.
7. Gupta AK, Jain HC, Lynde C (1997) Prevalence and epidemiology of unsuspected onychomycosis in patients visiting dermatologists' offices in Ontario, Canada--a multicenter survey of 2001 patients. Int J Dermatol 36: 783-787.

8. Haneke E, Roseeuw D (1999) The scope of onychomycosis: epidemiology and clinical features. Int J Dermatol 38: 7-12.

9. Catia MG, Fatima R (2010) Camil CasteloBranco.Menopause and aging: Changes in the immune system-A review. Maturitas 67: 316-320.

10. Calleja-Agius J, Brincat M, Borg M (2013) Skin connective tissue and ageing. Best Pract Res Clin Obstet Gynaecol 27: 727-740.

11. Pragya AN (2014) Dermatosis associated with menopause. J Midlife Health 5:168-175. 